Jaafari N, Bachollet MS, Paillot C, Amiel A, Rotge JY, Lafay N, Quentin S, Wassouf I, Camus V, Senon JL, ElHage W. Obsessive compulsive disorder in a patient with twiddler's syndrome. Pacing and Clinical Electrophysiology: PACE 2009;32(3):399-402. doi:10.1111/j.1540-8159.2008.02251.x

\title{
Obsessive Compulsive Disorder in a Patient with Twiddler's Syndrome
}

\author{
NEMATOLLAH JAAFARI, M.D.,* MARIE-SOPHIE BACHOLLET, * CELINE \\ PAILLOT, $\dagger, *$ ALAIN AMIEL, + NICOLAS LAFAY, M.D.,* SOLENE QUENTIN, * \\ LOUIS TANDONNET,* WISSAM EL HAGE, M.D., PH.D., §, \# and JEAN-LOUIS \\ SENON, M.D., PH.D. * \\ * Department of Psychiatry, Centre Hospitalier Henri Laborit, 86000 Poitiers, France \\ $\dagger$ Laboratoire EVACLIPSY, Université Paris X Nanterre, France \\ \$ Department of Cardiology, CHRU La Miletrie, 86000 Poitiers, France \\ ${ }_{\S}^{\$}$ INSERM U930 \& IFR135, Université François Rabelais, Tours, France \\ \# Clinique Psychiatrique Universitaire, CHRU de Tours, France
}

\begin{abstract}
Twiddler's or Twist syndrome is the twisting of pulse generators around themselves. It may result from mechanical manipulation that can induce the malfunction of the device. In this case, twiddler's syndrome resulted from compulsive checking of the device. The implantable cardioverter-defibrillator (ICD) triggered the development of an obsessive compulsive disorder (OCD). Two invasive procedures were required to replace the ICD. Psychiatric intervention prevented the recurrence of twiddler's syndrome in this patient for more than two years. We believe that pre-implant psychiatric assessment should be the rule.
\end{abstract}

Twiddler's syndrome, obsessive compulsive disorder, anxiety

\section{Background}

First described by Bayliss et al. in 1968 [1], twiddler's or twist Syndrome is the twisting of pulse generators around themselves. A total or partial movement of the pulse generators or even a rupture of the isolating material around the generators may be responsible for the malfunction of the device. The etiology of the syndrome is often considered to be a large subcutaneous pocket of abundant fatty tissue in obese subjects. It may affect a pacemaker or an implantable cardioverter-defibrillator (ICD) and can be easily diagnosed on a chest X-ray. Re-intervention consists in the replacement of the pulse generators and the creation of a tight generator pocket.

The present report focuses on its psychological causes in a case due to post-implant compulsive behavior, resulting in two interventions to replace the ICD. The essential features of obsessive-compulsive disorder (OCD) are recurrent obsessions and compulsions severe enough to be time-consuming and to cause marked impairment.

\section{Case History}

This 47-year-old man presented for the third time 16 months after implantation of an ICD. The device was implanted under the left pectoral owing to sustained ventricular fibrillation 10 days after a lower lateral basal myocardial infarction. The patient had experienced an acute myocardial infarction ten years earlier. He had a long history of smoking (20 cigarettes per day for 30 years) and was treated for dyslipidemia. His father had died from a myocardial infarction at the age of 56, when he was 8-years-old. The ICD had already been replaced 
twice. The investigation did not show any cardiac event that could explain the syndrome. The patient has felt fearful of receiving shocks since the implantation.

A psychiatric assessment was recommended when a third intervention became necessary. Although the patient had no personal psychiatric history, he had an obsessive personality but did not meet the DSM-IV-TR criteria for OCD [2]. He spent less than 30 minutes per day on compulsions and was obsessed with order and cleanliness. At home he often argued with his wife over how things should be in order. The assessment consisted of a psychiatric clinical interview, a structured diagnostic psychiatric interview using the MINI (Mini-International Neuropsychiatric Interview) [3] and the completion of the Y-BOCS (Yale-Brown Obsessive Compulsive Scale) [4]. The Y-BOCS is a scale of hetero-evaluation designed to assess OCD severity. It consists of two parts with a total score of 40 points. One part is used to evaluate obsessions (20 points) and the other part is designed to evaluate compulsions (20 points). On the Y-BOCS the patient had an obsessive sub-score of 6 and a compulsive sub-score of 10 , i.e. total score of 16/40, reflecting a moderate OCD, according to the cut-offs of the scale global score [5].

The episode of myocardial infarction that had occurred 10 years earlier was associated with intense pain and loss of consciousness. He described this traumatic experience as follows: "I started to feel a little pain at night, then around 4 a.m. it became intense and I died". Since then, any chest pain was always a prodrome of a myocardial infarction for him.

Since the implantation of the ICD, the patient had become anxious and had developed an OCD. He touched his chest several times a day (up to 50 times). In the beginning, this gesture "allowed [him] to diminish the pain linked to the operation, by the warmth of [his] hand" and also allowed him to check that the ICD was still there in place. Finally, he could not resist touching the defibrillator in a precise way, with his four fingers of the right hand hooked in the left arm-pit and his thumb on the defibrillator, which he moved from right to left. These compulsive actions explain how the ICD had turned on itself and the pulse generators had become twisted. He had tried to make sense of his compulsions: "I was afraid that the pain would become intense like the last time, when I died, or would make me die again. But, this time I was going to receive a shock." Briefly, this obsessive patient had developed an OCD, which manifested itself in the device checking for fear of death or receiving a shock.

The patient's cognitive construct was as follows:

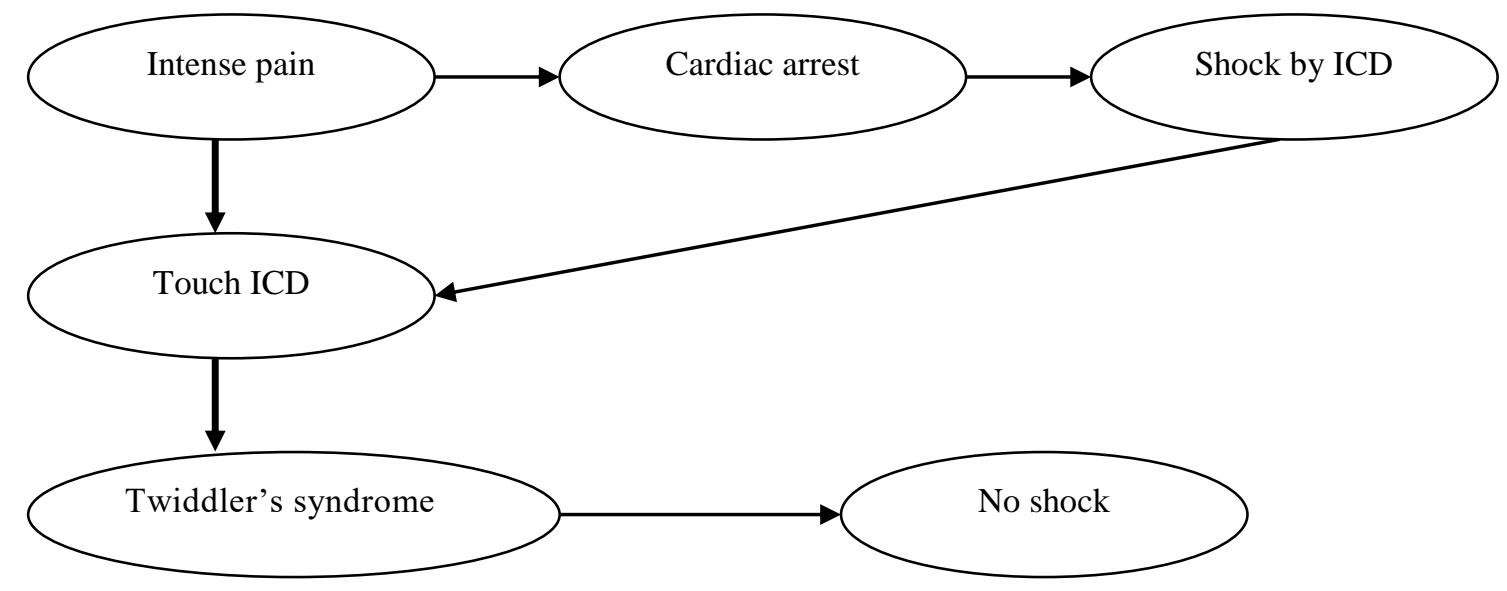


The patient received Cognitive Behavioral Therapy (CBT) involving exposure with response prevention (ERP) combined with an antidepressant (escitalopram $20 \mathrm{mg}$ per day) [6]. Exposure method consisted of prolonged exposure in imagination to the feared stimulus (anxious thoughts or image) and interoceptive exposure to chest pain until anxiety subsided. Moreover the patient was instructed no to engage in any checking behavior. This response prevention method aims at reality testing the dysfunctional danger schema that triggers compulsive behaviors. A qualified psychiatrist (NJ) administered individual CBT. Sessions took place for one hour once a week over 5 months, then twice a month during 6 months, with a total of 32 sessions over 11 months. Moreover, 15 sessions of Schultz relaxation training were provided over the same period.

No signs of recurrence of the syndrome were noted for 3 years. At the end of the therapeutic period, the Y-BOCS score dropped to 3/40 (i.e., no OCD according to the cutoffs of the score).

\section{Discussion}

This case clearly illustrates the role of compulsive device-checking behavior in the etiology of twiddler's syndrome. In this patient with an obsessive personality, the ICD became the trigger for his current OCD. Three defibrillators had to be replaced. CBT with relaxation sessions combined with an antidepressant treatment led to improvement of the OCD and avoided any relapse of the syndrome.

Twiddler's syndrome is considered a rare ailment, which occurs during the first year after insertion of the device. In a study of 4250 implants in 1989, Solti et al. [7] found 6 cases. In 1994, a review of the literature revealed only 10 reported cases [8]. However, some authors believe that the frequency of this syndrome is underestimated, which explains the delay in diagnosis and treatment. Viard [9] believes that this underestimation is due to the lack of movement of the pulse generators or to the rupture of the isolating material around the generators, but also to the fact that chest X-ray is not performed systematically. The syndrome usually occurs between 16 days [10,11] and 36 months [12] after insertion of the device. In this case it occurred 16 months after implantation. Typically, it concerns obese subjects, mostly women, with little muscular tissue and abundant subcutaneous fatty tissue, with a particular psychiatric background (e.g., generalized anxiety disorder). To date, two types of etiological factors have been established: mechanical [13-16] (large subcutaneous pocket in obese women with abundant fatty tissue [7, 10]) and psychiatric (manic syndrome) [7, 8, 17]. Neither of these etiologies was related to the insertion technique. This is the first report of $\mathrm{OCD}$ as an etiological factor.

Beauregard et al. [18] reported the case of a 44-year-old patient with an endocavitary pulse generator and a subcutaneous patch who, after losing $30 \mathrm{~kg}$ in weight, developed twiddler's syndrome, resulting in six shocks. Regarding the weight loss, the question is whether it was caused by a pre-existing psychiatric condition (i.e. depression, anxiety, eating disorders like anorexia, etc.) or was a reaction to the cardiac illness and therefore a transitory adjustment disorder. Furman [19] considered the syndrome to be more frequent when the stimulator pocket is larger than the apparatus. To prevent it, a Parsonnet Dacron pocket may be used. However, Avitall et al. [20] reported the occurrence of the syndrome despite using the pocket. Since fixing the stimulator to the bottom of a smaller pocket does not always prevent its occurrence [17], treating the psychiatric cause of the syndrome might be a promising alternative in preventing recurrence in some cases. 
Very often patients report not touching their device. This notion of the "unconscious or sub-conscious manipulation" has already been reported [8, 17]. The phenomenon could be related to the patient's "degree of insight", which is the level of recognition of their illness. Sometimes patients touch the apparatus in the early period following implantation, as an automatic reflex to alleviate the pain due to the scar and to check the integrity of the device as an adaptive behavior to the foreign object. Quite often, patients give it nicknames like (e.g., my baby, my toto, my rescuer...) in order to accept it psychologically. While the manipulations may stop quickly in patients with no psychiatric history and a good level of insight into their cardiac illness, they may continue in those suffering from a psychiatric disorder and with poor insight into their illness. In the present case, the patient had good insight, which suggests the need for systematic pre-implant psychiatric assessment and raising awareness about the ICD.

The latest defibrillators are smaller, more efficient and multifunctional. This reduction in size and weight seems to be one of the factors responsible for the onset of twiddler's syndrome, as reported by Crossley et al. [21]. They observed the case of a patient with a small ICD inserted in a retro-pectoral position who received inappropriate shocks due to the syndrome. They suggest that the small size in a retro-pectoral position and the discomfort caused by the device may be causal factors involved in the patient moving the device. However, the paper did not report any data on the personality or psychiatric history of the patient.

Twiddler's diagnosis is confirmed by a chest X-ray. Reinsertion is generally necessary with the replacement of the pulse generators and the creation of a smaller pocket. A recent study recommended regular chest X-ray follow-up to enable early diagnosis and treatment of twiddler's syndrome [22]. For others, systematic chest X-ray must be associated with a magnetic test on every patient equipped with a stimulator [9]. The present case also shows clearly that pre-implant psychiatric assessment is necessary. For patients with the syndrome, psychiatric assessment may also be necessary to determine the risk of an interfering psychiatric disorder. Anxiety and stress may lead to compulsive behavior, which represents a dysfunctional coping strategy, in patients with psychiatric syndromes or personality disorders. Hence, CBT such as stress management may reduce anxiety and have positive effects on stress-induced fibrillation [23, 24] and prevent twiddler's syndrome. This remains to be proved by systematic studies. In practice, psychiatric assessment [25] may be helpful to determine the global therapeutic strategy and particularly the usefulness of CBT in case of a psychiatric disorder such as OCD. Our case study suggests that psychological interventions may decrease the risk of the syndrome, especially when a psychiatric latent illness or a personality disorder is the etiology.

\section{Acknowledgments}

The authors thank Dr Jean Cottraux for providing advice on this article.

Conflicts of Interest: All other authors report no biomedical financial interests or potential conflicts of interest.

\section{Reference}

1. Bayliss CE, Beanlands DS, Baird RJ. The pacemaker-twiddler's syndrome: a new complication of implantable transvenous pacemakers. Can Med Assoc J 1968;99(8):371-373.

2. American Psychiatric Association. Diagnostic and Statistical Manual of Mental Disorders, fourth edition, revised (DSM-IV-TR). Washington DC, APA, 2000. 
3. Sheehan DV, Lecrubier Y, Sheehan KH, Amorim P, Janavs J, Weiller E, Hergueta T, et al. 1998. The Mini-International Neuropsychiatric Interview (M.I.N.I.): the development and validation of a structured diagnostic psychiatric interview for DSMIV and ICD-10. J Clin Psychiatry 59, Suppl 20, 22-33.

4. Mollard E, Cottraux J, Bouvard M. [French version of the Yale-Brown Obsessive Compulsive Scale]. Encephale 1989;15(3):335-341.

5. Bouvard M, Cottraux J. Protocoles et échelles d'évaluation en psychiatrie et en psychologie, quatrième édition. Paris, Masson, 2005, pp. 280.

6. Millet B, Jaafari N. Treatment of obsessive-compulsive disorder. Rev Prat 2007;57(1):53-57.

7. Solti F, Moravcsik E, Renyi-Vamos FJr, Szabo Z. Pacemaker twiddler's syndrome (rotation of the pacemaker around the electrode cable, a rare complication of pacemaker therapy). Acta Chir Hung 1989;30(3):231-236.

8. Newland GM, Janz TG. Pacemaker-twiddler's syndrome: a rare cause of lead displacement and pacemaker malfunction. Ann Emerg Med 1994;23(1):136-138.

9. Viard P. Le Twiddler's syndrome: à propos d'un cas. Stimucoeur 1989;2(26):82-84.

10. Khalilullah M, Khanna SK, Gupta U, Padmavati S. Pacemaker twiddler's syndrome: a note on its mechanism. J Cardiovasc Surg (Torino) 1979;20(1):95-100.

11. Fang CC, Tsai LM. Pacemaker twiddler's syndrome: report of a case. J Formos Med Assoc 1995;94(10):630-631.

12. Defaye P, Ormezzano O, Deharo JC, Denis B. [A rare cause of inappropriate shocks of an implantable automatic defibrillator. Twiddler syndrome]. Arch Mal Coeur Vaiss 1997;90(7):999-1002.

13. Chernilas JZ, Bilfinger TV, Vlay SC. Pacemaker Twiddler's syndrome. Pacing Clin Electrophysiol 1996;19(12 Pt 1):2158-2159.

14. Asbach S, Brunner M, Zehender M, Bode C, Faber TS. Multiple inappropriate defibrillator discharges due to Twiddler's syndrome. Wien Klin Wochenschr 2005;117(23-24):801.

15. Occhetta E, Bortnik M, Francalacci G, Magnani A, Plebani L, Vassanelli C. Twiddler's syndrome and pectoral stimulation in a patient with dual-site unipolar atrial pacing. Ital Heart J 2004;5(5):399-402.

16. Vural A, Agacdiken A, Ural D, Komsuoglu B. Reel syndrome and pulsatile liver in a patient with a two-chamber pacemaker. Jpn Heart J 2004;45(6):1037-42.

17. Lal RB, Avery RD. Aggressive pacemaker twiddler's syndrome. Dislodgement of an active fixation ventricular pacing electrode. Chest 1990;97(3):756-757.

18. Beauregard LA, Russo AM, Heim J, Snyder H, Waxman HL. Twiddler's syndrome complicating automatic defibrillator function. Pacing Clin Electrophysiol 1995;18(4 Pt 1):735-737.

19. Furman S. Defibrillator twiddler's syndrome. Ann Thorac Surg 1995;59(2):544-546.

20. Avitall B, Stormo A, Barragry T, Axtel K, Hare J. Sudden cardiac death: Twiddler's syndrome with an implantable cardioverter defibrillator. Am Heart J 1994;128(4):833836.

21. Crossley GH, Gayle DD, Bailey JR, Haisty WK, Simmons TW, Fitzgerald DM. Defibrillator twiddler's syndrome causing device failure in a subpectoral transvenous system. Pacing Clin Electrophysiol 1996;19(3):376-377.

22. Larrouse E, Rodríguez E, Moya AA, Rodríguez O, Soler Soler J. [Twiddler's syndrome in a patient with implantable cardioverter defibrillator: an avoidable complication?]. Rev Esp Cardiol 2001;54(12):1456-1458. 
23. Chevalier P, Cottraux J, Mollard E, Sai N, Brun S, Burri H, Restier L, et al. Prevention of implantable defibrillator shocks by cognitive behavioral therapy: a pilot trial. Am Heart J 2006;151(1):191.

24. Chevalier P, Verrier P, Kirkorian G, Touboul P, Cottraux J. Improved appraisal of the quality of life in patients with automatic implantable cardioverter defibrillator: a psychometric study. Psychother Psychosom 1996;65(1):49-56.

25. Jaafari N, Serra W, Cormier C, Lafay N, Senon JL. Dépression et maladies coronariennes. Médecine thérapeutique 2006;12(2):79-88. 